\title{
Monitoring and sudden infant death syndrome: an update
}

\author{
Report from the Foundation for the Study of Infant Deaths and \\ the British Paediatric Respiratory Group
}

Apnoea monitors and sudden infant death were the subject of a report prepared for the Foundation for the Study of Infant Deaths and the British Paediatric Respiratory Group in $1985 .^{1}$ The evidence for and against respiratory monitoring and guidelines for the management and support of families issued with monitors were given. The five conclusions of that article were: (i) proof that some infants have a period of apnoea before irreversible brain damage occurs (the hypothesis underlying the use of the devices) is lacking; (ii) none of the respiratory monitors currently available is entirely satisfactory; (iii) the risk of recurrence of sudden infant death in a family is small; (iv) close supervision of, and support for, the families of infants being monitored is essential; and (v) apnoea monitoring does have a place, but only in selected circumstances.

Public awareness of the sudden infant death syndrome (SIDS) has continued to increase in the past few years; so too have parental demands and publicity for the use of respiratory monitors at home. Some of the more relevant work that has been published about monitoring and sudden infant death since the 1985 report is summarised in 'question and answer' form below.

Is there now evidence to suggest that there is a period of apnoea before death in SIDS?

The precise mode(s) of death in SIDS remain uncertain and unrecorded. Similarities between SIDS and apparently life threatening events for example, (near miss SIDS or apparent lifelessness) in which children may be found apnoeic, cyanotic or pale, and hypotonic, have been described. These episodes have various causes, mainly associated with the respiratory, cardiac, and central nervous systems, though the provoking factor of some remains undiagnosed. Apnoea during apparently life threatening events may be central, obstructive, or mixed. ${ }^{2-5}$ The distribution of intrathoracic petechiae and blotchy haemorrhages found at necropsy in many SIDS cases has been interpreted as a sign of negative intrathoracic pressure suggestive of upper airway obstruction. ${ }^{6}$ Functional collapse of the pliable pharyngeal airway during life will not be detected after death.

These apparently life threatening events occur in the same age range as SIDS and have the same male:female ratio. Many epidemiological characteristics of the two groups of infants are also similar. Infants with apparently life threatening events do not, however, show the seasonal distribution of SIDS, and the episodes occur significantly less often between 2000 and 0800 hours. ${ }^{4}$ In the only population survey that we know of, nearly a third of the infants had further apnoeic episodes within three days of the first, but only $12 \%$ had a recurrence later. ${ }^{3}$ The inference must be that had the more serious episodes been unobserved sudden death could have resulted; it did occur in a small proportion later. Less than $7 \%$ of SIDS cases, however, had previous histories of apparently life threatening events. ${ }^{1}$

\section{Can infants who are at increased risk of SIDS} now be identified?

The known epidemiological risk factors for SIDS (like physiological factors) are not considered significantly sensitive or specific, even in combination, to identify infants for particular intervention. $^{7}$

There is no recent population based data for this country on recurrence rates among siblings. Figures from the United States, ${ }^{8}$ Norway, ${ }^{9}$ and Australia ${ }^{10}$ have recently been published. When matched for mother's age and birth order, the rate of SIDS in subsequent siblings of babies who died of SIDS in the state of Washington did not differ from that of controls. ${ }^{8}$ When Norwegian data were similarly adjusted for mothers age and parity, the relative risk for further sudden unexpected infant deaths was 2.8. ${ }^{9}$ In South Australia Beal and Blundell found that for most families (92\%) the risk of recurrence was less than twice the expected risk. They identified a small subgroup (8\%) with a significantly increased risk. ${ }^{10}$

Are more satisfactory monitors now available? The monitors used at home in Britain are usually respiratory monitors, which detect only central apnoea. Cardiorespiratory monitors have been more widely used in Europe and the United States to detect the bradycardia that may accompany obstructive apnoea.

The Consensus Development Conference listed 12 'essential' and eight 'desirable' characteristics for impedance cardiorespiratory monitors. ${ }^{11}$ The best available models fully met only seven, and partially met only two, of the essential characteristics. The three that were not met were the detection of obstructive and mixed apnoea and a minimum of false positive, or false 
negative, alarms. None of the eight desirable characteristics were fully met, and only two were partially met. The 1985 statement on monitors ${ }^{1}$ listed the Graseby Dynamics MR10 Respiration Monitor, the Vickers Apnoea Alarm Mk 3 and mattress, the Eastwood Eastleigh Apnoea Respiration Monitor RE 200 (battery), RE 134 Apnoea Monitor (pressure pad) (mains), and the impedance systems (for example Healthdyne, Airshields, and Hewlett Packard). Their comparative advantages and disadvantages were described.

It was pointed out that none reliably identified cessation of respiratory air flow, including obstructive apnoea; all gave false alarms, and none incorporated a data storage system that would allow analysis of events preceding an alarm. The Graseby MR10, which is activated by abdominal movements, has been most widely used. The Densa monitor is similar in its applications, and its sensor is housed in an elastic belt that can be applied over clothing and can be easily moved without changing adhesive tapes on the skin if the position is wrong. The newer Densa monitor incorporates a supplementary alarm facility for minimum number of breaths/minute; this is aimed at the detection of irregular gasps that may occur during obstructive breathing efforts, but again relies primarily on abdominal movements. Rapid obstructive breathing efforts could not be expected to activate the alarms on either the Graseby or Densa monitors.

All impedance monitors are more expensive than other forms of breathing movement detectors. More recent Healthdyne models coordinate alarms that are activated if breathing movements and heart rate coincide at low rates, so avoiding failure of the alarm if there is a forceful cardiac impulse during prolonged central apnoea. Although theoretically less likely, body movements and some partially obstructed breathing efforts may still activate the respiratory channel in individual infants. A modified 'respiration index' detector system is being promoted as part of an alternative impedance monitor recently introduced as Atlas AR800 by the British Technology Group; although theoretically a useful advance, large trials are still required. The necessary application of electrodes to skin, and dependence on AC power supply, are potential disadvantages.

The detection of hypoxaemia by monitors has only recently been considered for use at home. Though transcutaneous oxygen monitors are unsuitable for long term unsupervised use outside hospital, pulse oximetry has the advantages that the probe needs less frequent changing and reliability is less affected by skin texture. Overheating and pressure necrosis of the skin have been described, however, and reliability in active infants is still under study. Poor quality of pulse signals during movement of the probe on a limb may compromise the monitor's usefulness in measuring both oxygen saturation and pulse rate during episodes of obstructive breathing. The Nellcor device with simultaneously recorded electrocardiogram seems to reduce the incidence of false alarms, but the necessary application of chest electrodes is a disadvantage. The oximeter most commonly used in the United Kingdom is the Ohmeda.

An ambitious scheme of monitoring has been developed by Johnson et al in the United Kingdom. It is 'based on pulse oximetry and inductance plethysmography, but (is) also able to detect body movements and temperatures (body and ambient) and accept signals from measurements of transcutaneous or respired $\mathrm{CO}_{2}$ or $\mathrm{O}_{2}$, and pneumotachograph, ECG and EMG measurement for more detailed studies'. This can sample, partly analyse, and store data, which can then be transferred by telephone to a computer so that there is a two way link. ${ }^{12}$ It is being used experimentally in the home.

Acoustic monitors designed to detect breath sounds, and to register apnoea if they are absent, have been described but are impractical for clinical use as are airflow detectors placed in the upper airway. Video recorders, infrared movement detectors, and other equipment used as monitors in research studies are also impractical because they require constant supervision, are too complicated for use at home, or are prohibitively expensive.

What is known about the beneficial or harmful effects of monitoring at home?

Many families who have had a sudden, unexpected, and unexplained infant death are made less anxious after the birth of a subsequent child if a respiratory monitor is available as part of a comprehensive support service; for others the devices increase anxiety. Adequately conducted studies on the psychological effects of monitoring are few, but the results suggest that some stress is always present. ${ }^{13-15}$ This important aspect needs more careful study of larger numbers of families. The 1985 statement, ${ }^{1}$ and the recent United States National Institutes of Child Health and Human Development sponsored Consensus Development Conference on infantile apnoea and home monitoring, ${ }^{16}$ emphasised that adequate professional support services for families using home monitors were essential.

Which infants should be monitored at home? Infants who have been found apnoeic, cyanosed or pale, and hypotonic-an apparently life threatening event-are possible candidates. The decision will depend on the nature of the provoking event (if identified), and this too may suggest the need for specific treatment, the most useful form of monitoring, or both. If no cause is found, the issue of a respiration monitor often remains a subject for discussion between the parents and paediatricians.

The families of subsequent siblings of infants who died of SIDS may often be helped by a comprehensive scheme of alternative support. A small controlled trial assessed support measures for 100 self selected families of such infants. All of the families kept charts of the symptoms and had weekly visits from a health visitor. In addition either respiratory monitors or scales for daily weighing were allocated randomly. Both systems proved acceptable, but support from 
and discussion about the infants with health professionals was a vital aspect of management. ${ }^{17}$ The Foundation for the Study of Infant Deaths is now funding a scheme that it hopes may ensure that all families receive adequate support with the care of such infants. The purpose of this programme (CONI, Care of the Next Infant) is to establish such support in districts where it is lacking, and improve its organisation in districts where it is available. It is hoped that the scheme will gradually become autonomous.

The conclusions reached by Milner in 1985 have not been substantially altered by recent research. ${ }^{1}$ A respiratory monitor that relies on movement to detect apnoea such as the Densa or Graseby MR10 will detect absence of breathing movements more reliably and earlier than human observation alone. These devices cannot reliably detect obstructive apnoea and evidence currently available suggests that this is more likely to be a possible mode of sudden unexpected death than prolonged central apnoea. Conductance pad monitors (Eastwood RE 134, Eastleigh RE 200) of similar cost avoid attachments to the baby and may suit some families or infants better. It must be re-emphasised that the decision to use a monitor at home should be accompanied by the provision of adequate support for the families, instruction on the use of the device, and instruction on how to resuscitate the infant.

We thank Dr U MacFadyen for her contribution to the section giving details of monitors.

1 Milner AD. Apnoea monitors and sudden infant death. Report from the Foundation for the Study of Infant Deaths and the British Paediatric Respiratory Group. Arch Dis Child 1985;60:76-80.

2 Simpson H, MacFadyen UM. Near-miss for sudden infant death syndrome: a clinical approach. In: Meadow R, ed. Recent advances in paediatrics. 1986;8:201-16.

3 Wennergren G, Milerad J, Lagercrantz H, et al. The epidemiology of sudden infant death syndrome and attacks of lifelessness in Sweden. Acta Paediatr Scand 1987;76: 898-906.

4 Kahn A, Blum D, Hennart P, et al. A critical comparison of the history of sudden-death infants and infants hospitalised for near-miss SIDS. Eur F Pediatr 1984;143:103-7.

5 Southall DP, Talbert DG. Mechanisms for abnormal apnoea of possible relevance to the sudden infant death syndrome. Ann NY Acad Sci 1988;533:329-49.

6 Beckwith JB. The mechanism of death in sudden infant death syndrome. In: Culbertson JL, Krous HF, Bendell RD, eds. Sudden infant death syndrome. Medical aspects and psychological management. London: Edward Arnold, 1989: $48-61$.

7 Froggatt P, Beckwith JB, Schwartz PJ. Valdes-Dapena M, Southall DP. Cardiac and respiratory mechanisms that might be responsible for sudden infant death syndrome. Ideas for future research. Ann NY Acad Sci 1988;533: 421-6.

8 Peterson DR, Sabotta EE, Daling JR. Infant mortality among subsequent siblings of infants who died of sudden infant death syndrome. $\mathcal{F}$ Pediatr 1986;108:911-4.

9 Irgens LM, Skjaerven R. Sudden infant death syndrome and post perinatal mortality in Norwegian birth cohorts post perinatal mortality in Norwegian birth

10 Beal SM, Blundell HK. Recurrence incidence of sudden infant death syndrome. Arch Dis Child 1988;63:924-30.

11 Report of a Consensus Development Conference. Infantile apnea and home monitoring. Washington: US Department of Health and Human Services, 1987.

12 Johnson P, Sands P, Head J, Stott FD, Humphreys S. A new on-line ambulatory monitoring system for studying cardiorespiratory development in infants at home. Ann NY Acad Sci 1988;53:459-60.

13 Lyman RD, Wurtele SK, Wilson DR. Psychological effects on parents of home and hospital apnea monitoring. f Pediatr Psychol 1985;10:439-48.

14 Desmarez C, Blum D, Montauk L, Kahn A. Impact of home monitoring for sudden infant death syndrome on family life. A controlled study. Eur $\mathcal{F}$ Pediatr 1987;146:159-61.

15 Wasserman AL. A prospective study of the impact of home monitoring on the family. Pediatrics 1984;74:323-9.

16 Consensus Statement. National Institutes of Health consensus development conference on infantile apnea and home monitoring, Sept 29 to Oct 1, 1986. Pediatrics 1987;79: 292-9.

17 Emery JL, Waite AJ, Carpenter RG, Limerick SR, Blake D. Apnoea monitors compared with weighing scales for siblings after cot death. Arch Dis Child 1985;60:1055-60. 